\title{
Nanostructured surfaces for nuclear astrophysics studies in laser-plasmas
}

\author{
Carmen Altana ${ }^{1}$, Vincenzo Amarù ${ }^{2}$, Giuseppe Castro ${ }^{1}$, Marco Frassetto ${ }^{3,4}$, Gaetano \\ Lanzalone $^{1,5}$, Luciana Malferrari $^{3}$, David Mascali ${ }^{1}$, Annamaria Muoio $^{1}$, Fabrizio Odorici $^{3}$ \\ and Salvo Tudisco ${ }^{1}$ \\ ${ }^{1}$ INFN - LNS, Via S. Sofia 62, 95123 Catania, Italy \\ ${ }^{2}$ Univ. degli Studi di Catania, Via Santa Sofia, 95123 Catania, Italy \\ ${ }^{3}$ INFN- Sez. di Bologna, Viale B. Pichat, 6/2, 40127 Bologna, Italy \\ ${ }^{4}$ Dip. di Fisica e Astronomia, Univ. degli Studi di Bologna, V.le B. Pichat 6/2, 40127 Bologna, Italy \\ ${ }^{5}$ Univ. degli Studi di Enna "Kore", Via delle Olimpiadi, 94100 Enna, Italy
}

\begin{abstract}
The future availability of high-intensity laser facilities capable of delivering tens of petawatts of power (e.g. ELI-NP) into small volumes of matter at high repetition rates will give the unique opportunity to investigate nuclear reactions and fundamental interactions process under extreme plasma conditions [1]. In this context, use of targets with nanostructured surfaces is giving promising indications to reproduce plasma conditions suitable for measurements of thermonuclear reactions rates, in the domain of nanosecond laser pulses.
\end{abstract}

\section{Introduction}

Plasma plays a primary role in modern astrophysics since it constitutes the $99 \%$ of the matter in the Universe. Therefore, the investigation of the origin of stars and related phenomena deals closely with plasma physics. Direct and indirect measurements of the relevant nuclear cross sections have been performed over the years by using accelerated ion beams [2-5] and innovative experimental apparatus [6,7].

Although it is impossible to reproduce the extreme properties of stellar matter, keV temperature plasmas can be rather easily generated in laboratory through magnetic confinement, but with densities orders magnitude lower than the stellar ones. An improvement on this direction can be achieved by using Laser-plasmas [8-10]. In the last decades, laser fabrication technology has evolved rapidly and further new physics has emerged from the study of Laser-plasmas [11-15].

In such contest, in order to achieve conditions of astrophysical interest, suitable for nuclear fusion, the application of nano-technologies in this field has also been investigated. Nanostructures on the targets surface, based on metallic nanowires (NWs) embedded in porous alumina [16], can be useful since they can absorb light at very high efficiency. These expectations are confirmed from experiments in different laser pulses regimes (from $\mathrm{ns}$ to fs) and various intensities $[17,18]$.

Moreover, these nanostructures allow to incorporate deuterium into the solid matrix, in order to produce laser deuterated-plasmas. This contribution will focus on the preliminary 
results of an experiment that aims to produce such plasmas, to study $\mathrm{D}+\mathrm{D}$ reaction rates in the laboratory, in a condition that is closer to that of the stars.

\section{Experimental Set-up}

A Q-switched Nd:YAG laser operating at $1064 \mathrm{~nm}$, with a maximum energy of $2 \mathrm{~J}$ and a duration of $6 \mathrm{~ns}$ impinges on a target at an intensity of about $4 \cdot 10^{12} \mathrm{~W} / \mathrm{cm}^{2}$, with an angle of $30^{\circ}$ with respect to the beam. The target is placed inside an interaction chamber, where a pressure of about at $10^{-}$Torr is kept. The chamber, as sketched in Fig. 1a), can be equipped with several diagnostics, in order to characterize the plasma and the nuclear products.

An ICCD for optical fast imaging, placed perpendicularly to the plasma expansion direction, allows investigating the spatio-temporal evolution and velocities of plasmas in the visible domain. A cooled CCD camera, placed at $60^{\circ}$ from the target normal axis, sensitive to soft X-rays, is a fundamental diagnostic to characterize the X-ray plasma emission. This camera is equipped with a pinhole array: each pinhole is filtered by using Al-made attenuators of increasing thickness, ranging from 3 to $20 \mu \mathrm{m}$, in order to suppress low energy photons and to select X-rays at increasing energies. Another configuration consists of a single Al-layer, $50 \mu \mathrm{m}$ thick, in order to operate in single photon counting for measuring the X-ray energy spectra and extrapolating plasma temperatures [19]. Ions collectors and $\mathrm{SiC}$ detectors [20-23] operating in Time-of-Flight configuration, are placed in the forward direction respect to the plasma propagation, in order to reconstruct the velocity distribution of charged particles escaped from the plasma. A further device is a Thomson Parabola Spectrometer, which allows the identification of ions species and their charge state distribution besides the ability to extract the energy distribution [24-26]. In order to detect and identify neutrons from $\mathrm{D}+\mathrm{D}$ reactions, the final set-up will include also an array of plastic scintillators with a Silicon Photo-multiplier read-out [27].

In the experiment, we used targets produced at the INFN lab in Bologna [16]. In order to compare performances of nanowires with different composition, identical alumina matrices with the same diameter $(20 \mathrm{~nm})$ and thickness $(10 \mu \mathrm{m})$, were filled with cobalt, iron and nickel. A sample of bulk aluminum was also irradiated, to be used as reference. Since the different nanostructured targets provide similar results in terms of plasma temperature, only the Co-nanowires target analysis is included in this contribution.

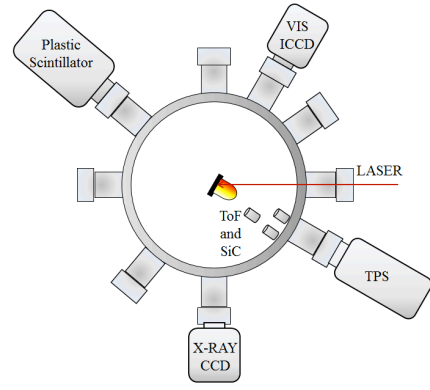

a)

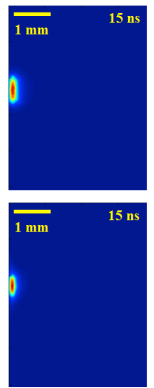

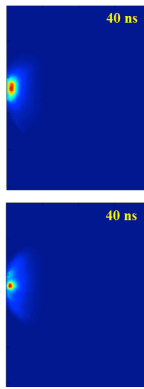

b)
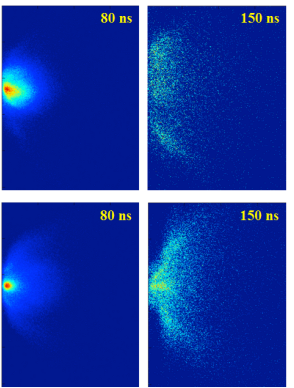

)

Fig. 1. a) Schematic view of the experimental setup; b) sequence of ICCD images, showing the spatio-temporal evolution of bulk Al (top) and Co-nanostructured (bottom) plume at various delays.

\section{Results}

Fig. 1b) shows typical time-resolved ICCD images for bulk Al and Co-nanostructured targets. All images are spectrally integrated in the region $200-700 \mathrm{~nm}$ and normalized to 
their maximum intensity in order to easily compare them. Colours indicate different radiation intensities: higher counts (red) correspond to areas with higher temperature and particle density in the plasma plume. It can be observed that plasma duration of nanostructured target is higher than that of bulk Al: at $150 \mathrm{~ns}$ Co-nanostructured plume intensity is about $50 \%$ higher than bulk Al. By studying the expansion velocity of the plasma, through the fit of the position-time images of the luminous front, we observe that for bulk Al the plume has a higher velocity than for nanostructured targets, where plasma evolves more slowly and has a longer life. This could be an evidence of plasma stagnation, which implies a hotter and denser plasma.

Furthermore, a previous analysis of the X-ray emission has evidenced an increasing of $\mathrm{X}$-ray flux for nanostructured targets compared to bulk $\mathrm{Al}$ and quite similar temperature due to a cold plasma component, extracted by fitting the fluxes with an opportune procedure, as described in [28]. A new set of measurements with a Co-nanowires target, performed by using the X-CCD in Single Photon Counting configuration, has allowed to obtain the X-ray spectra and to provide a more precise estimation of the plasma temperature [19]. Fig. 2a) shows the as-detected X-ray spectrum. The plasma temperature, extracted by fitting the spectrum in the range 4.5-6 keV, after deconvolution for CCD quantum efficiency and Al-filter transmission (see the insert), corresponds to $0.72 \pm 0.14 \mathrm{keV}$.

Moreover, we estimated that deuterium can be incorporated in the alumina matrix (via physical-chemical processes) with a density of about $10^{20}$ atoms $/ \mathrm{cm}^{3}$, which allow investigating the $\mathrm{D}+\mathrm{D}$ reaction in a plasma environment. We calculated the expected fusion rates in our experimental conditions, as in [9], using the cross-section from D+D scattering data and assuming that deuterons have a Maxwellian energy distribution, having the same temperature as our plasma. Results are shown in Fig 2b), where the number of fusion events in a single shot is calculated, by assuming plasma temperatures that vary from $100 \mathrm{eV}$ to 1 $\mathrm{keV}$. In particular, the highlighted region represents our experimental condition, with a temperature of $0.72 \pm 0.14 \mathrm{keV}$ : in that range, the fusion events in a single shot are close to one. This is a promising result for the next aim to study $\mathrm{D}+\mathrm{D}$ reactions in plasmas.

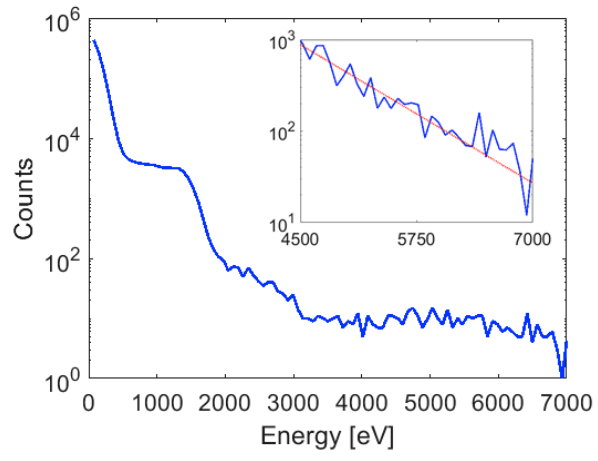

a)

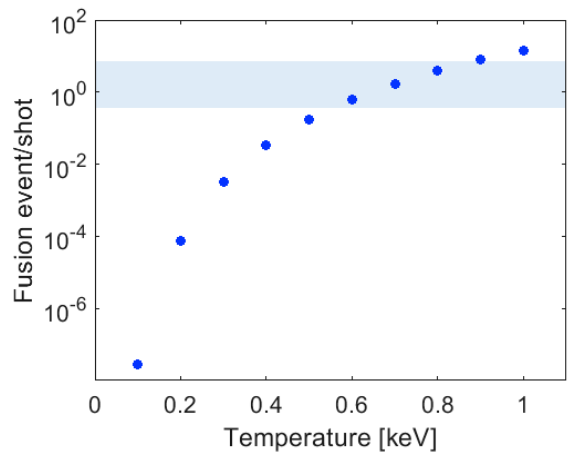

b)

Fig. 2. a) As-detected $X$-ray spectrum for a Co-nanowires target; the insert shows the fit to the spectrum, after deconvolution for detection efficiencies, in the range 4.5-6 keV; b) expected fusion rate in a single shot, as a function of the plasma temperature.

\section{Conclusions}

A preliminary characterization of plasmas generated during Nd:YAG laser ablation in ns domain from nanostructured targets has been obtained. Different targets, based on Co, $\mathrm{Fe}$ and Ni NWs, show similar results in terms of visible and X-ray emission. 
By observing the plasma expansion, through time-resolved ICCD images of the visible emission, we find experimental evidence to deem that stagnant plasmas with high temperature and density can be obtained by means of nanostructured materials. Such result is in agreement with the X-ray emission characterization. Although the X-ray energy is similar for bulk Al and NWs targets, a higher X-ray intensity was observed for all nanostructured samples compared to bulk $\mathrm{Al}$ : this indicates an increasing in terms of plasma density and/or in terms of plasma stagnation for NWs targets. Moreover, operating in proper conditions, we estimated that a significant number of fusion events could be obtained by means of nanostructured targets containing deuterium. This scenario could be promising since it could allow studying specific nuclear reactions in plasmas for astrophysical applications.

\section{References}

1. Negoita, F., et al. Rom. Rep. Phys. 68, (2016)

2. La Cognata, M., et al. J. Phys. G Nucl. Part. Phys., 35 (2007)

3. Cherubini, S., et al. Eur. Phys. J. A, 20 (2004)

4. La Cognata, M. et al. Phys. Lett. B, 664 (2008)

5. Tumino, A., et al. Nucl. Phys. A, 718 (2003)

6. Del Zoppo, A., et al. Nucl. Instrum. Methods Phys. Res. A, 581 (1996)

7. Cardella, G., et al. Nucl. Instrum. Methods Phys. Res. A, 378 (1996)

8. Mascali, D., et al. Radiat. Eff. Defect. S, 165 (2010)

9. Mascali, D., et al. Proc. Int. Astron. Union, 6 (2010)

10. Ivanovski, S. L. et al., Radiat. Eff. Defect. S, 165 (2010)

11. Mascali, D., et al. Eur. Phys. Lett., 100 (2012)

12. Tudisco, S., et al. Nucl. Instrum. Methods Phys. Res. A, 653 (2011)

13. Gambino, N., et al. Appl. Surf. Sci., 272 (2013)

14. Tudisco, S., et al. Rev Sci Instrum., 87 (2016)

15. Gambino, N., et al. Radiat. Eff. Defect. S, 165 (2010)

16. Malferrari, L., et al. J.Nanotechnol., (2009) 149691

17. Lanzalone, G., et al. Rev. Sci. Instrum., 87 (2016)

18. Cristoforetti, G., et al. Plasma Phys. Contr. F. Journal, 56 (2014)

19. Mascali, D., et al. Rev. Sci. Instrum., 85 (2014)

20. Sciacca, E., et al. IEEE Photonics Technol. Lett., 18 (2006)

21. Mazzillo, M., et al. Sens. Actuators A Phys., 138 (2007)

22. Privitera, S., et al. Sensors, 8 (2008)

23. Muoio, A., et al. EPJ Web of Conferences 117 (2016)

24. Schillaci, F., et al. J. Instrum., 9 (2014)

25. Muoio, A., et al. Nucl. Instrum. Methods Phys. Res. A, 829 (2016)

26. Altana, C., et al. Rev. Sci. Instrum., 87 (2016)

27. Finocchiaro, P., et al. IEE Trans. Nucl. Sci., 52 (2005)

28. Muoio, A., et al. J. Instrum., 12 (2017) 